\title{
Piecemeal Retrograde Removal of Encrusted and Encased Stuck Ureteral Stent: Video Tips and Tricks
}

\section{Amelia Pietropaolo, MD, FEBU}

Department of Urology, University Hospital Southampton NHS Foundation Trust, Southampton, United Kingdom.

E-mail: ameliapietr@gmail.com

\section{Lily Whitehurst, MD}

Department of Urology, University Hospital Southampton NHS Foundation Trust, Southampton, United Kingdom.

\section{Bhaskar K. Somani, MD}

Department of Urology, University Hospital Southampton NHS Foundation Trust, Southampton, United Kingdom.

() Mary Ann Liebert, Inc. DOI: 10.1089/vid.2019.0057
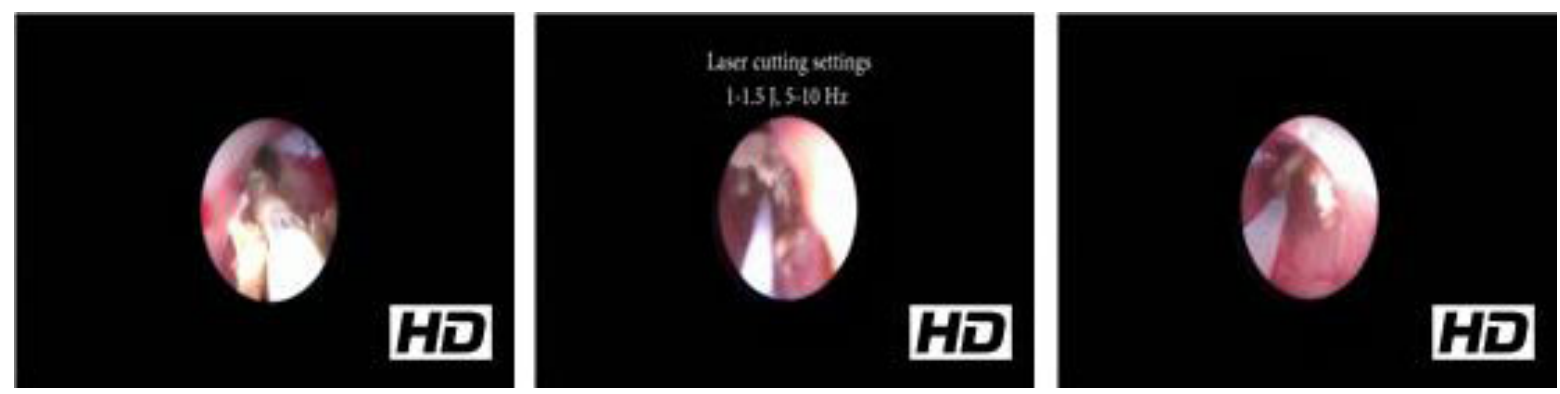

\section{Abstract}

Introduction: Ureteral stent encrustation leads to morbidity related to ureteral obstruction along with urinary tract infection or sepsis. The time duration it takes for stent encrustation is variable, but the risk $^{1}$ increases with stent dwell time. Removal of encrusted stent where these encrustations completely ${ }^{2}$ encase the stent can be extremely challenging. In this video we show a case of complete stent encasement where we used a piecemeal technique to remove the stent through retrograde ureteroscopy (URS) and laser incision. ${ }^{3}$

Materials and Methods: Three cases of piecemeal removal of encrusted encased stents were performed. The stents were heavily encrusted, and a retrograde URS under a general anesthesia was performed with a laser to cut the stent, with piecemeal removal of the pieces. Cutting the stent allowed to create space to improve visibility and mobility in the ureter, as the ureteroscope progressed proximally, freeing and removing the distal cut portion of the stent.

Results: The removal of stent using a cystoscopic grasper was not possible because of heavy encrustation associated with it, encasing the stent. A cystoscopic-guided safety wire was inserted to the kidney. A storz semirigid ureteroscope was then inserted parallel to the stent. Owing to stent encasement and lack of space to navigate the ureteroscope proximally, the distal end of the stent was cut by a laser. Using the ureteroscope, the encrustations around the stent were then laser fragmented $(0.4-0.6 \mathrm{~J}, 5-10 \mathrm{~Hz})$ using the Ho:YAG laser to gradually release the encrustations. The stent was then divided using the cutting setting $(1-1.5 \mathrm{~J}, 5-10 \mathrm{~Hz})$. This allowed to create space and a gradual step-by-step proximal progression of the ureteroscope to release the next segment of the stent. The cut fragments of the stent were progressively removed using Ngage basket. The process was repeated to allow complete removal of the stent retrogradely. We had to divide the stent several 
times to finally release and remove it. The procedure was carried out effectively and no intra- or postoperative complications were noted. The piecemeal removal of stent strategy was effective in all three patients. A vigilant approach and early intervention should be adopted in patients with a high risk $^{4}$ of stent encrustation, which includes patients with recurrent urinary tract infections, urease producing bacteria, history of cystine or uric acid stone, and avoiding prolonged stent dwell time. Most encrusted stents can be removed intact but in severe encrustations that encase the stent completely and intact removal is not possible, then piecemeal removal might be the only way to remove the stent endoscopically. Stent material, design, and coatings all influence biofilm formation and stent encrustations, and this should be borne in mind while choosing it for a given patient.

Conclusion: Piecemeal stent removal is necessary in extreme cases of stent encrustation where it is encasing the stent completely. This approach allowed effective retrieval of stents in all cases, which otherwise may have needed percutaneous or an open surgical approach.

No competing financial interests exist.

All authors contributed equally in writing this article.

Runtime of video: 2 mins 35 secs

Keywords: stent, encrustations, ureter, laser, cut

\section{Cite this video}

Amelia Pietropaolo, Lily Whitehurst, Bhaskar K. Somani, Piecemeal Retrograde Removal of Encrusted and Encased Stuck Ureteral Stent: Video Tips and Tricks, Videourology. 2020,

DOI: $10.1089 / v i d .2019 .0057$.

\section{References}

1. Kawahara T, Ito T, Terao H, Yoshida M, Matsuzaki J. Ureteral stent encrustation, and colouring: Morbidity related to indwelling times. J Endourol 212;26:178-182.

2. Aravantionos e, Gravas S, Karatzas AD, Tzortzis V, Melekos M. Forgotten, encrusted ureteral stents: A challenging problem with an endourologic solution. J Endourol 2006;20:1045-1049.

3. Acosta-Miranda AM, Milner J, Turk TM. The FECal double-J: A simplified approach in the management of encrusted and retained ureteral stents. J Endourol 2009;23:409-415.

4. Thomas A, Cloutier J, Villa L, Letendre J, Ploumidis A, Traxer O. Prospective analysis of a complete retrograde ureteroscopic technique with holmium laser stent cutting for management of encrusted ureteral stents. J Endouol 2017;31:476-481.

Original Publication Date: 2020 